\title{
Automated Unit Test Generation for Python
}

\author{
Stephan Lukasczyk, Florian Kroiß, and Gordon Fraser \\ University of Passau, Innstr. 33, 94032 Passau, Germany \\ \{stephan.lukasczyk, gordon.fraser\}@uni-passau.de, kroiss@fim.uni-passau.de
}

\begin{abstract}
Automated unit test generation is an established research field, and mature test generation tools exist for statically typed programming languages such as Java. It is, however, substantially more difficult to automatically generate supportive tests for dynamically typed programming languages such as Python, due to the lack of type information and the dynamic nature of the language. In this paper, we describe a foray into the problem of unit test generation for dynamically typed languages. We introduce PYNGUIN, an automated unit test generation framework for Python. Using Pynguin, we aim to empirically shed light on two central questions: (1) Do well-established search-based test generation methods, previously evaluated only on statically typed languages, generalise to dynamically typed languages? (2) What is the influence of incomplete type information and dynamic typing on the problem of automated test generation? Our experiments confirm that evolutionary algorithms can outperform random test generation also in the context of Python, and can even alleviate the problem of absent type information to some degree. However, our results demonstrate that dynamic typing nevertheless poses a fundamental issue for test generation, suggesting future work on integrating type inference.
\end{abstract}

Keywords: Dynamic Typing - Python - Random Test Generation . Whole Suite Test Generation

\section{Introduction}

Unit tests can be automatically generated to support developers and the dynamic analysis of programs. Established techniques such as feedback-directed random test generation [15] or evolutionary algorithms $[7]$ are implemented in mature research prototypes, but these are based on strong assumptions on the availability of static type information, as is the case in statically typed languages like Java. Dynamically typed languages such as Python or JavaScript, however, have seen increased popularity within recent years. Python is the most popular programming language in the category of dynamically typed languages, according to, for example, the IEEE Spectrum Ranking ${ }^{1}$ It is heavily used in the fields of machine learning and data analysis, and it is also popular in other domains. This

\footnotetext{
1 https://spectrum.ieee.org/computing/software/the-top-programming-languages-2019 accessed 2020-07-25.
} 
can be seen, for example, from the Python Package Index (PyPI), which contains more than 200000 packages at the time of writing. In languages like Python, the type information that automated unit test generators require is not available.

An automated unit test generator primarily requires type information in order to select parameters for function calls and to generate complex objects. If type information is absent, the test generator can only guess which calls to use to create new objects, or which existing objects to select as parameters for new function calls. Existing test generators for dynamically typed languages therefore resort to other means to avoid having to make such choices in the first place, for example by using the document object model of a web browser to generate tests for JavaScript [14], or by targetting the browser's event handling system rather than APIs [3, 12]. However, there is no general purpose unit test generator at API level yet for languages like Python.

In order to allow test generation research to expand its focus from statically to dynamically typed languages, in this paper we introduce Pynguin, a new automated test generation framework for Python. PYNGUin takes as input a Python module and its dependencies, and aims to automatically generate unit tests that maximise code coverage. In order to achieve this, PYNGUIN implements the established test generation techniques of whole-suite generation 9 and feedback-directed random generation [15]. PYNGUiN is available as open source to support future research on automated test generation for dynamically typed programming languages. PyNGUIN is designed to be extensible; in this paper we focus on established baseline algorithms for foundational experiments, we will add further algorithms such as DynaMOSA [16] in future work.

Using Pynguin, we empirically study the problem of automated unit test generation for Python using ten popular open source Python projects taken from GitHub, all of which contain type information added by developers in terms of type annotations. This selection allows us to study two central questions: (1) Do previous findings, showing that evolutionary search achieves higher code coverage than random testing [5, also generalise to dynamically typed languages? (2) What is the influence of the lack of type information in a dynamically typed language like Python on automated unit test generation?

In detail, the contributions of this paper are the following:

1. We introduce Pynguin, a new framework for automated unit test generation for the Python programming language.

2. We replicate experiments previously conducted only in the context of statically typed languages to compare test generation approaches.

3. We empirically study the influence of type information on the effectiveness of automated test generation.

Our experiments confirm that the whole-suite approach generally achieves higher code coverage than random testing, and that the availability of type information also leads to higher resulting coverage. However, our experiments reveal several new technical challenges such as generating collections or iterable input types. Our findings also suggest that the integration of current research on type inference is a promising route forward for future research. 
In both scenarios, type information is crucial: In the forward construction type information is used to inform the choice of call to append to the sequence, while in the backward construction type information is used to select generators of dependency objects. Without type information, which is the case with the Python example, a forward construction (1) has to allow all possible functions at all steps, thus may not only select the constructor of Bar, but also that of Foo with an arbitrary parameter type, and (2) has to consider all existing objects for all parameters of a selected call, and thus, for example, also str or int. Backwards construction without type information would also have to try to select generators from all possible calls, and all possible objects, which both result in a potentially large search space to select from.

Type information can be provided in two ways in recent Python versions: either in a stub file that contains type hints or directly annotated in the source code. A stub file can be compared to $\mathrm{C}$ header files: they contain, for example, method declarations with their according types. Since Python 3.5, the types can also be annotated directly in the implementing source code, in a similar fashion known from statically typed languages (see PEP $484^{2}$.

\section{Search-based Unit Test Generation}

\subsection{Python Test Generation as a Search Problem}

As the unit for unit test generation, we consider Python modules. A module is usually identical with a file and contains definitions of, for example, functions, classes, or statements; these can be nested almost arbitrarily. When the module is loaded the definitions and statements at the top level are executed. While generating tests we do not only want all definitions to be executed, but also all structures defined by those definitions, for example, functions, closures, or list comprehensions. Thus, in order to apply a search algorithm, we first need to define a proper representation of the valid solutions for this problem.

We use a representation based on prior work from the domain of testing Java code 9 . For each statement $s_{j}$ in a test case $t_{i}$ we assign one value $v\left(s_{j}\right)$ with type $\tau\left(v\left(s_{j}\right)\right) \in \mathcal{T}$, with the finite set of types $\mathcal{T}$ used in the subject-undertest (SUT) and the modules imported by the SUT. We define four kinds of statements: Primitive statements represent int, float, bool, and str variables, for example, $\operatorname{var} 0=42$. Value and type of a statement are defined by the primitive variable. Note that although in Python everything is an object, we treat these values as primitives because they do not require further construction in Python's syntax. Other simple types, such as lists, require the construction of the list and its elements, which we do not yet handle. Constructor statements create new instances of a class, for example, $\operatorname{var} 0=$ SomeType () . Value and type are defined by the constructed object; any parameters are satisfied from the set $V=\left\{v\left(s_{k}\right) \mid 0 \leq k<j\right\}$. Method statements invoke methods on objects, for example, $\operatorname{var} 1=\operatorname{var} 0 . f \circ o()$. Value and type are defined by the return value

\footnotetext{
2 https://python.org/dev/peps/pep-0484/, accessed 2020-07-25.
} 
of the method; source object and any parameters are satisfied from the set $V$. Function statements invoke functions, for example, $\operatorname{var} 2=\operatorname{bar}()$. They do not require a source object but are otherwise identical to method statements. This representation is of variable size; we constrain the size of test cases $l \in[1, L]$ and test suites $n \in[1, N]$. In contrast to prior work on testing Java [9], we do not define field or assignment statements; fields of objects are not explicitly declared in Python but assigned dynamically, hence it is non-trivial to identify the existing fields of an object and we leave it as future work.

The search operators for this representation are based on those used in EvoSuite [9]: Crossover takes as input two test suites $P_{1}$ and $P_{2}$, and generates two offspring $O_{1}$ and $O_{2}$. Individual test cases have no dependencies between each other, thus the application of crossover always generates valid test suites as offspring. Furthermore, the operator decreases the difference in the number of test cases between the test suites, thus abs $\left(\left|O_{1}\right|-\left|O_{2}\right|\right) \leq \operatorname{abs}\left(\left|P_{1}\right|-\left|P_{2}\right|\right)$. Therefore, no offspring will have more test cases than the larger of its parents.

When mutating a test suite $T$, each of its test cases is mutated with probability $\frac{1}{|T|}$. After mutation, we add new randomly generated test cases to $T$. The first new test case is added with probability $\sigma$. If it is added, a second new test case is added with probability $\sigma^{2}$; this happens until the $i$-th test case is not added (probability: $1-\sigma^{i}$ ). Test cases are only added if the limit $N$ has not been reached, thus $|T| \leq N$. The mutation of a test case can be one of three operations: remove, which removes a statement from the test case, change, which randomly changes values in a statement - for example, by adding random values to numbers, adding/replacing/deleting characters, or changing method calls - and insert, which adds new statements at random positions in the test case. Each of these operations can happen with the same probability of $\frac{1}{3}$. A test case that has no statements left after the application of the mutation operator is removed from the test suite $T$. For constructing the initial population, a random test case $t$ is sampled by uniformly choosing a value $r$ with $1 \leq r \leq L$, and then applying the insertion operator repeatedly starting with an empty test case $t^{\prime}$, until $\left|t^{\prime}\right| \geq r$.

\subsection{Covering Python Code}

A Python module contains various control structures, for example, if or while statements, which are guarded by logical predicates. The control structures are represented by conditional jumps at the bytecode level, based on either a unary or binary predicate. We focus on branch coverage in this work, which requires that each of those predicates evaluates to both true and false.

Let $B$ denote the set of branches in the SUT - two for each conditional jump in the byte code. Everything executable in Python is represented as a code object. For example, an entire module is represented as a code object, a function within that module is represented as another code object. We want to execute all code objects $C$ of the SUT. Therefore, we keep track of the executed code objects $C_{T}$ as well as the minimum branch distance $d_{\min }(b, T)$ for each branch $b \in B$, when executing a test suite $T . B_{T} \subseteq B$ denotes the set of taken branches. We then define the branch coverage $\operatorname{cov}(T)$ of a test suite $T$ as $\operatorname{cov}(T)=\frac{\left|C_{T}\right|+\left|B_{T}\right|}{|C|+|B|}$. 
The fitness function required by the genetic algorithm of our whole-suite approach is constructed similar to the one used in EvoSuite 9 by incorporating the branch distance. Branch distance is a heuristic to determine how far a predicate is away from evaluating to true or false, respectively. In contrast to previous work on Java, where most predicates at the bytecode level operate only on Boolean or numeric values, in our case the operands of a predicate can be any Python object. Thus, as noted by Arcuri [2], we have to define our branch distance in such a way that it can handle arbitrary Python objects.

Let $\mathbb{O}$ be the set of possible Python objects and let $\Theta:=\{\equiv, \not \equiv,<, \leq,>, \geq, \in$ $, \notin,=, \neq\}$ be the set of binary comparison operators (remark: we use ' $\equiv$ ', '=', and ' $\in$ ' for Python's $==$, is, and in keywords, respectively). For each $\theta \in \Theta$, we define a function $\delta_{\theta}: \mathbb{O} \times \mathbb{O} \rightarrow \mathbb{R}_{0}^{+} \cup\{\infty\}$ that computes the branch distance of the true branch of a predicate of the form $a \theta b$, with $a, b \in \mathbb{O}$ and $\theta \in \Theta$. By $\delta_{\bar{\theta}}(a, b)$ we denote the distance of the false branch, where $\bar{\theta}$ is the complementary operator of $\theta$. Let further $k$ be a positive number, and let $\operatorname{lev}(x, y)$ denote the Levenshtein distance [11 between two strings $x$ and $y$. The predicates is numeric $(z)$ and is_string $(z)$ determine whether the type of their argument $z$ is numeric or a string, respectively.

$$
\begin{aligned}
& \delta_{\equiv}(a, b)= \begin{cases}0 & a \equiv b \\
|a-b| & a \neq \equiv b \wedge \text { is_numeric }(a) \wedge \text { is_numeric }(b) \\
\operatorname{lev}(a, b) & a \neq \equiv b \wedge \text { is_string }(a) \wedge \text { is_string }(b) \\
\infty & \text { otherwise }\end{cases} \\
& \delta_{<}(a, b)= \begin{cases}0 & a<b \\
a-b+k & a \geq b \wedge \text { is_numeric }(a) \wedge \text { is_numeric }(b) \\
\infty & \text { otherwise }\end{cases} \\
& \delta_{\leq}(a, b)= \begin{cases}0 & a \leq b \\
a-b+k & a>b \wedge \text { is_numeric }(a) \wedge \text { is_numeric }(b) \\
\infty & \text { otherwise }\end{cases} \\
& \delta_{>}(a, b)=\delta_{<}(b, a) \\
& \delta_{\geq}(a, b)=\delta_{\leq}(b, a) \\
& \delta_{\theta}(a, b)=\left\{\begin{array}{ll}
0 & a \theta b \\
k & \text { otherwise }
\end{array} \quad \theta \in\{\not \equiv, \in, \notin,=, \neq\}\right.
\end{aligned}
$$

Note that every object in Python represents a Boolean value and can therefore be used as a predicate. We assign a distance of 0 to the true branch of such a unary predicate, if the object represents a true value, otherwise $k$. Future work shall refine the branch distance for different operators and operand types.

The fitness function estimates how close a test suite is to covering all branches of the SUT. Thus, every predicate has to be executed at least twice, which we 
enforce in the same way as existing work 9 : the actual branch distance $d(b, T)$ is given by

$$
d(b, T)= \begin{cases}0 & \text { if the branch has been covered } \\ \nu\left(d_{\min }(b, T)\right) & \text { if the predicate has been executed at least twice } \\ 1 & \text { otherwise }\end{cases}
$$

with $\nu(x)=\frac{x}{x+1}$ being a normalisation function 9 .

Finally, we can define the resulting fitness function $f$ of a test suite $T$ as

$$
f(T)=|C|-\left|C_{T}\right|+\sum_{b \in B} d(b, T)
$$

\subsection{The PYNGUIN Framework}

PYNGUiN is a framework for automated unit test generation written in and for the Python programming language. The framework is available as open-source software licensed under the GNU Lesser General Public License from its GitHub repository ${ }^{3}$ It can also be installed from the Python Package Index (PyPI) ${ }^{\sqrt{4}}$ using the pip utility.

Pynguin takes as input a Python module and allows the generation of unit tests using different techniques. For this, it parses the module and extracts information about available methods in the module and types from the module and its imports. So far, PyNGUIN focuses on test-input generation and excludes the generation of oracles. A tool run emits the generated test cases in the style of the widely-used PyTest 5 framework or for the unittest module from the Python standard library.

PYNGUiN is built to be extensible with other test generation approaches and algorithms. For experiments in this paper, we implemented a feedback-directed random approach based on RANDOOP 15 in addition to the whole-suite testgeneration approach. Feedback-directed test generation starts with two empty test suites, a passing and a failing test suite, and adds statements randomly to an empty test case. After each addition, the test case is executed and the execution result is retrieved. Successful test cases, that is, test cases that do not raise exceptions are added to the passing test suite; a test case that raises an exception is added to the failing test suite. In the following, the algorithm randomly chooses a test case from the passing test suite or an empty test case and adds statements to it. We refer the reader to the description of RANDOOP [15] for details on the algorithm; the main differences of our approach are that it does not yet check for contract violations, and does not require the user to provide a list of relevant classes and methods, which RANDOOP does.

\footnotetext{
3 https://github.com/se2p/pynguin, accessed 2020-07-27.

4 https://pypi.org/project/pynguin/, accessed 2020-07-25.

5 https://www.pytest.org, accessed 2020-07-25.
} 


\section{Experimental Evaluation}

Using our PYNGUIN test generator, we aim to empirically study automated unit test generation on Python. First, we are interested in determining whether previous findings on the performance of test generation techniques established in the context of statically typed languages generalise also to Python:

Research Question 1 (RQ1) How do whole-suite test generation and random test generation compare on Python code?

A central difference between prior work and the context of Python is the type information: Previous work evaluated test-generation techniques mainly for statically typed languages, such as Java, where information on parameter types is available at compile time, that is, without running the program. This is not the case for many programs written in dynamically typed languages, such as Python. Therefore, we want to explicitly evaluate the influence of the type information for the test-generation process:

Research Question 2 (RQ2) How does the availability of type information influence test generation?

\subsection{Experimental Setup}

In order to answer the two research questions, we created a dataset of Python projects for experimentation. We used the 'typed' category of the PyPI package index of Python projects, and selected ten projects by searching for projects that contain type hints in their method signatures, and that do not have dependencies to native-code libraries, such as numpy. Details of the chosen projects are shown in Table 1 the column Project Name gives the name of the project on PyPI; the lines of code were measured with the $\mathrm{CLOC}^{6}$ utility tool. The table furthermore shows the absolute average number of code objects, predicates, and detected types per module of each project. The former two measures give an insight on the project's complexity; higher numbers indicate larger complexity. The latter provides an overview how many types PyNGUiN was able to parse (note that Pynguin may not be able to resolve all types).

The central metric we use to evaluate the performance of a test generation technique is code coverage. In particular, we measure branch coverage at the level of bytecode; like in Java bytecode, complex conditions are compiled to nested branches with atomic conditions also in Python code. In addition to the final overall coverage, we also keep track of coverage over time to shed light on the speed of convergence. In order to statistically compare results we use the Mann-Whitney U-test and the Vargha and Delaney effect size $\hat{A}_{12}$.

We executed PyNGUin in four different configurations: First, we executed PYNGUIN using random test generation and whole test suite generation; second, we ran PYNGUin with the developer-written type annotations contained in the

\footnotetext{
6 https://github.com/AlDanial/cloc, accessed 2020-07-25.
} 
Table 1: Projects used for evaluation

\begin{tabular}{lrrrrrr}
\hline Project Name & Version & LOCs & Modules & CodeObjs. & Preds. & Types \\
\hline apimd & 1.0 .2 & 316 & 1 & 35.0 & 83.0 & 11.0 \\
async_btree & 1.0 .1 & 284 & 6 & 9.0 & 8.7 & 6.3 \\
codetiming & 1.2 .0 & 85 & 2 & 18.0 & 8.0 & 6.0 \\
docstring_parser & 0.7 .1 & 608 & 6 & 12.0 & 15.7 & 9.5 \\
flutes & 0.2 .0$. post0 & 1085 & 9 & 19.0 & 26.0 & 5.0 \\
flutils & 0.6 & 1715 & 13 & 10.2 & 22.3 & 8.4 \\
mimesis & 4.0 .0 & 1663 & 34 & 12.3 & 5.7 & 9.2 \\
pypara & 0.0 .22 & 1305 & 6 & 47.2 & 23.5 & 12.0 \\
python-string-utils & 1.0 .0 & 476 & 4 & 21.0 & 29.5 & 6.5 \\
pytutils & 0.4 .1 & 1108 & 23 & 8.2 & 6.6 & 6.1 \\
\hline Total & & 8645 & 104 & 191.9 & 229.0 & 79.9 \\
\hline
\end{tabular}

projects, and without them. To answer RQ1, we compare the performance of random test generation and whole test suite generation; to answer RQ2 we compare the performance of each of these techniques for the case with and without type information.

For each project, PYNGUin was run on each of the constituent modules in sequence. We executed PyNGUIN in GIT revision 5f538833 in a Docker container that is based on Debian 10 and utilises Python 3.8.3. In line with previous work, we set the maximum time limit for the test-generation algorithms, that is, the time without analysing the module-under-test and without export of the results, to $600 \mathrm{~s}$ per module. We ran PYNGUin 30 times on each module and configuration to minimise the influence of randomness. All experiments were executed on dedicated compute servers equipped with Intel Xeon E5-2690v2 CPUs and 64 GB RAM, running Debian 10. All scripts and the raw data are available as supplementary materia/7.

\subsection{Threats to Validity}

Internal Validity The standard coverage tool for Python is Coverage.Py, which offers the capability to measure branch coverage. However, it measures branch coverage by comparing which transitions between source lines have occurred and which are possible. This method of measuring branch coverage is imprecise, because not every branching statement necessarily leads to a source line transition, for example, $\mathrm{x}=0$ if $\mathrm{y}>42$ else 1337. We thus implemented our own coverage measurement. We tried to mitigate possible errors in our implementation, by providing sufficient unit tests for it.

External Validity We used 104 modules from ten different Python projects for our experiments. It is conceivable that the exclusion of projects without type

\footnotetext{
7 https://github.com/se2p/artifact-pynguin-ssbse2020, accessed 2020-07-27.
} 


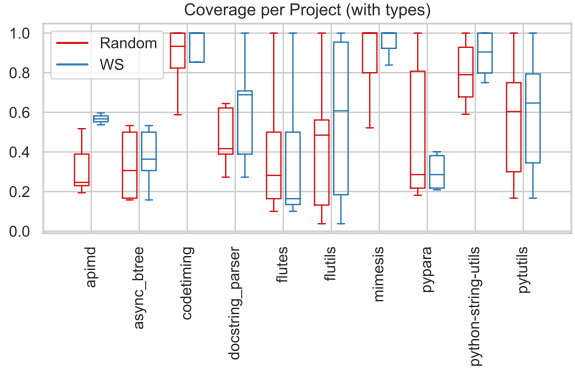

(a) With type information

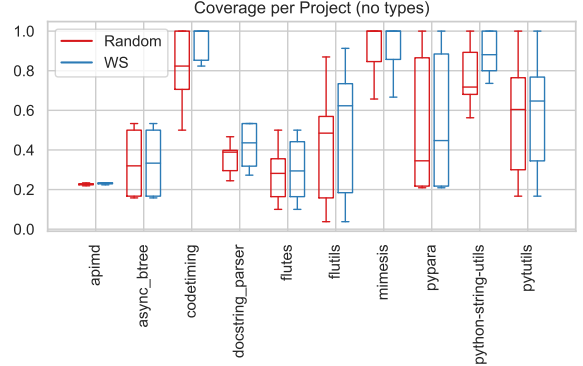

(b) Without type information

Figure 1: Coverage per project and configuration

annotations or native-code libraries leads to a selection of smaller projects, and the results may thus not generalise to other Python projects. However, besides the two constraints listed, no others were applied during the selection.

Construct Validity Methods called with wrong input types may still cover parts of the code before possibly raising exceptions due to the invalid inputs. We conservatively included all coverage in our analysis, which may improve coverage for configurations that ignore type information, and thus reduce the effect we observed. However, it does not affect our general conclusions. Further, we cannot measure fault finding capability as our tool does not generate assertions, which is explicitly out of scope of this work.

\subsection{RQ1: Whole-suite Test Generation vs. Random Testing}

Figure 1 provides an overview over the achieved coverage per project in box plots. Each data point in the plot is one achieved coverage value for one of the modules of the project. Figure 1a reports the coverage values for whole-suite and random test generation with available type hints, whereas Fig. $1 \mathrm{~b}$ reports the same without the usage of type hints to guide the generation.

Coverage values range from $0 \%$ to $100 \%$ depending on the project. The coverage achieved varies between projects, with some projects achieving generally high coverage (for example, python-string-utils, mimesis, codetiming), and others posing challenges for PYNGUIN (for example, apmid, async_btree, pypara, flutes). For example, for the apimd project without type information the coverage is slightly above $20 \%$, which is the coverage achieved just by importing the module. In Python, when a module is imported, the import statements of the module, as well as class and method definitions are executed, and thus covered. Note that this does not execute the method bodies. For other projects with low coverage, Pynguin is not able to generate reasonable inputs, for example, higher-order functions or collections, due to technical limitations. 


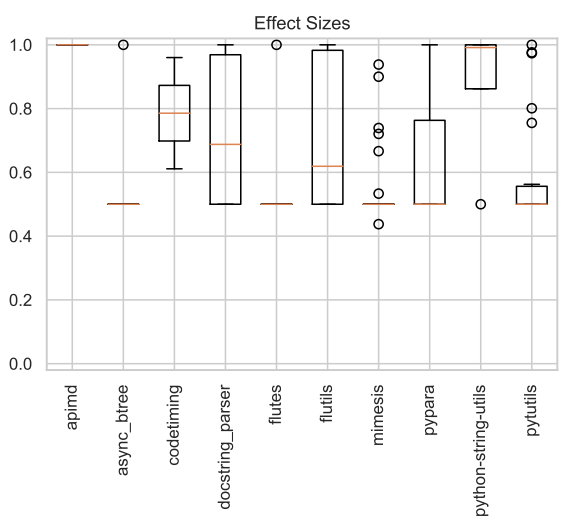

(a) $\hat{A}_{12}$ effect sizes with type information

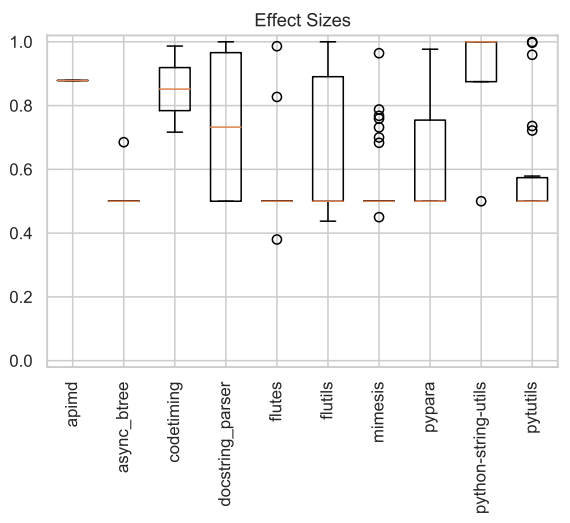

(b) $\hat{A}_{12}$ effect sizes without type information

Figure 2: Effect sizes of whole suite versus random generation. Values greater than 0.500 indicate whole suite is better than random.

To better understand whether whole-suite test generation performs better than random test generation, Fig. 2a reports the $\hat{A}_{12}$ effect sizes for the permodule comparison of the two with available type information, whereas Fig. $2 \mathrm{~b}$ reports the same without available type information. In both box plots, a value greater than 0.500 means that whole-suite performs better than random test generation, that is, yields higher coverage results. Both plots show that on average whole-suite does not perform worse than random and, depending on the project, is able to achieve better results in terms of coverage (average $\hat{A}_{12}$ with type information: 0.618 , without type information: 0.603). The effect of these improvements is significant $(p<0.05)$ for six out of ten projects, most notably for apimd $\left(\hat{A}_{12}=1.00, p\right.$-value $\left.<0.001\right)$, python-string-utils $\left(\hat{A}_{12}=\right.$ $0.705, p$-value $<0.001)$, and codetiming $\left(\hat{A}_{12}=0.636, p\right.$-value $\left.=0.00531\right)$.

For the other projects the effect is negligible. In case of mimesis $\left(\hat{A}_{12}=0.530\right)$ this is due to high coverage values in all configurations - most method parameters expect primitive types, which are also used for input generation if no type information is given. Other projects require specific technical abilities, for example, most methods in async_btree $\left(\hat{A}_{12}=0.535\right)$ are coroutines, which require special calls that cannot currently be generated by PYNGUin. The consequence of this technical limitations is that PYNGUIN cannot reach higher coverage independent of the used algorithm in these cases. We observed that methods under test often require collection types as inputs, in Python prevalently lists and dictionaries. Also generating these input types would allow us to execute more parts of the code which would lead to higher coverage and thus better results. We leave this, however, as future work.

A further current limitation of our framework lies in how the available type information is processed. PYNGUIN can currently only generate inputs for concrete 


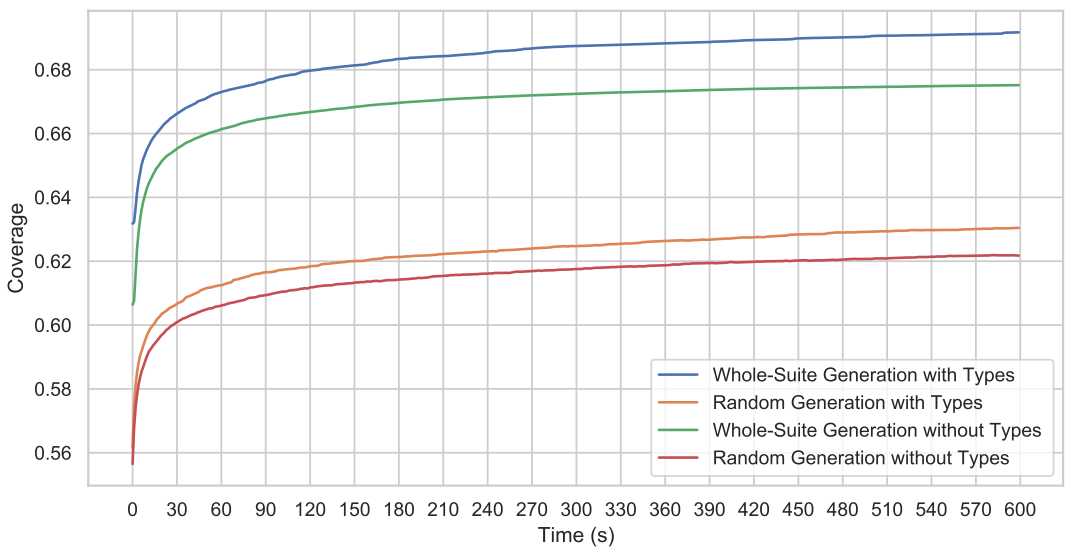

Figure 3: Average coverage development over time

types, unions of types, and the Any type - for which it attempts to use a random type from the pool of available types in the SUT. Future work shall handle sub-typing relations as well as generic types 10 . Another prevalent parameter type that limits our current tool are callables, that is, higher order functions that can be used, for example, as call backs. Previous work has shown that generating higher-order functions as input types is feasible for dynamically typed languages and beneficial for test generation 18. Furthermore, Pynguin currently only has a naive static seeding strategy for constants that incorporates all constant values from the project under test into test generation, whereas seeding has been show to have a positive influence on the quality of test generation [8] since it allows better-suited input values.

Figure 3 shows the development of the average coverage over all modules over the available generation time of $600 \mathrm{~s}$. The line plot clearly indicates that whole-suite generation achieves higher coverage than random generation, which again supports our claim. Overall, we can answer our first research question as follows:

Summary (RQ1): Whole-suite test generation achieves at least as high coverage as random test generation. Depending on the project it achieves moderate to strongly higher coverage.

\subsection{RQ2: Influence of Type Information}

To answer RQ2 we compare the coverage values between the configurations with and without type annotations, again using the per-module $\hat{A}_{12}$ effect sizes on the coverage values. This time, we show the effect of type information for whole-suite generation in Fig. 4a and for random generation in Fig. 4b. For whole-suite generation, we observe a large positive effect on some modules, and barely any 


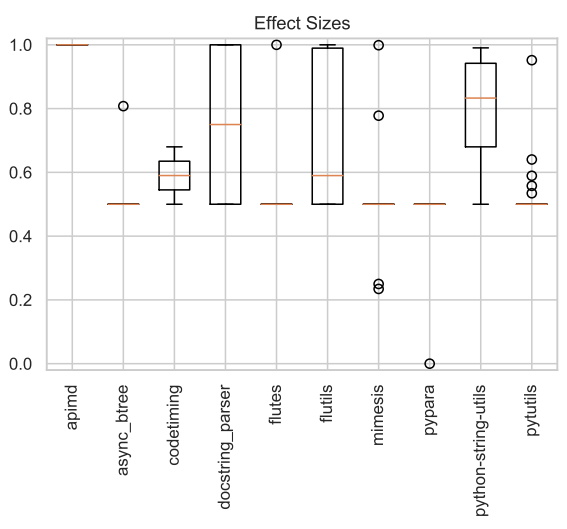

(a) $\hat{A}_{12}$ effect sizes for whole-suite generation

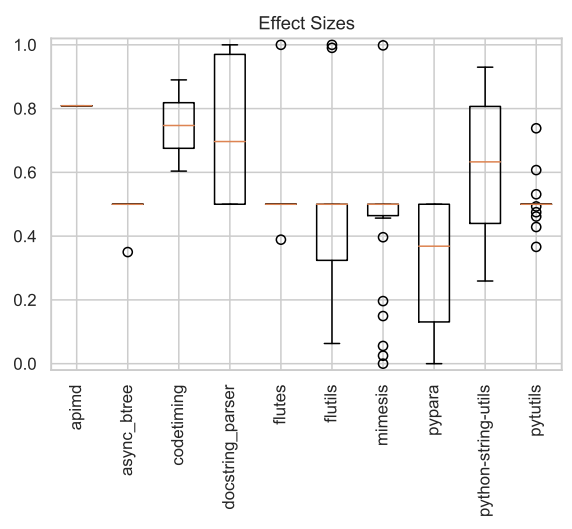

(b) $\hat{A}_{12}$ effect sizes for random generation

Figure 4: Effect sizes for type influences

effect for other modules when type information is incorporated; we report an average $\hat{A}_{12}$ value of 0.578 in favour of type information. For random generation, we note similar effects, except for the pypara project $\left(\hat{A}_{12}=0.411, p\right.$-value $=$ $0.00937)$; inspecting the pypara source code reveals that it uses abstract-class types as type annotations. PYNGUIN tries to instantiate the abstract class, which fails, and is thus not able to generate method inputs for large parts of the code because it cannot find an instantiable subtype. Overall, however, we report an average $\hat{A}_{12}$ value of 0.554 in favour of random generation with type information.

The box plots in Fig. 4 indicate similar conclusions for both whole-suite and random testing: the availability of type information is beneficial for some projects while its effect is negligible for other projects. The docstring_parser project, for example, requires their own custom types as parameter values for many methods. Without type information, PYNGUIN has to randomly choose types from all available types, with a low probability of choosing the correct one, whereas with available type information it can directly generate an object of correct type. Another effect comes in place for the python-string-utils projects: most of its methods only require primitive input types but very specific input values. PYNGUIN utilises a simple static constant seeding heuristic for input-value generation. Due to many values in the constant pool the chance of picking the correct value is smaller when not knowing the requested type, thus leading to lower coverage without type information.

On the other hand, projects such as flutes require iterables and callables as parameters in many cases or need special treatment of their methods to execute them properly (see coroutines in async_btree, for example). PYNGUIN currently lacks support to generate these required types, which prevents larger effects but does not limit the general approach. Thus, the type information cannot be used effectively, which results in negligible effects between the compared configurations. 
The line plot in Fig 3 shows the average coverage per evaluated configuration over the available time for test generation. It shows that both for whole-suite and random generation the configuration that incorporates type information yields higher coverage values over the full runtime of the generation algorithms, compared to ignoring type information. This again supports our claim that type information is beneficial when generating unit tests for Python programs. Overall, we therefore conclude for our second research question:

Summary $(\boldsymbol{R Q} 2)$ : Incorporating type information supports the test generation algorithms and allows them to cover larger parts of the code. The strength of this effect, however, largely depends on the SUT. Projects that require specific types from a large pool of potential types benefit more, and thus achieve larger effect sizes, than projects only utilising simple types.

\section{Related Work}

Closest to our work is whole-suite test generation in EvoSuiTe [9] and feedbackdirected random test generation in RANDOOP [15]. Both of these approaches target test generation for Java, a statically typed language, whereas our work adapts these approaches to Python.

To the best of our knowledge, little has been done in the area of automated test generation for dynamically typed languages. Approaches such as SYMJS 12 or JSEFT [14] target specific properties of JavaScript web applications, such as the browser's DOM or the event system. Feedback-directed random testing has also been adapted to web applications with ARTEMIS [3]. Recent work proposes LAMBDATESTER [18], a test generator that specifically addresses the generation of higher-order functions in dynamic languages. Our approach, in contrast, is not limited to specific application domains.

For automated generation of unit tests for Python we are only aware of AUGER 8 it generates test cases from recorded SUT executions, while our approach does the generation automatically.

\section{Conclusions}

In this paper we presented PyNGuin, an automated unit test generation framework for Python that is available as an open source tool, and showed that PyNGUin is able to emit unit tests for Python that cover large parts of existing code bases. PYNGUIN provides a whole-suite and a random test generation approach, which we empirically evaluated on ten open source Python projects. Our results confirm previous findings from the Java world that a whole-suite approach can outperform a random approach in terms of coverage. We further showed that the availability of type information has an impact on the test generation quality. Our investigations revealed a range of technical challenges for automated test

\footnotetext{
8 https://github.com/laffra/auger, accessed 2020-07-25.
} 
generation, which provide ample opportunities for further research, for example, the integration of further test-generation algorithms, such as (Dyna)MOSA [16], the generation of assertions, or the integration of type inference approaches.

\section{References}

1. Andrews, J.H., Menzies, T., Li, F.C.: Genetic algorithms for randomized unit testing. IEEE Trans. Software Eng. 37(1), 80-94 (2011)

2. Arcuri, A.: It really does matter how you normalize the branch distance in searchbased software testing. Softw. Test. Verification Reliab. 23(2), 119-147 (2013)

3. Artzi, S., Dolby, J., Jensen, S.H., Møller, A., Tip, F.: A framework for automated testing of JavaScript web applications. In: Proc. ICSE. pp. 571-580. ACM (2011)

4. Baresi, L., Miraz, M.: Testful: Automatic unit-test generation for java classes. In: Proc. ICSE. vol. 2, pp. 281-284. ACM (2010)

5. Campos, J., Ge, Y., Albunian, N., Fraser, G., Eler, M., Arcuri, A.: An empirical evaluation of evolutionary algorithms for unit test suite generation. Inf. Softw. Technol. 104, 207-235 (2018)

6. Csallner, C., Smaragdakis, Y.: Jcrasher: an automatic robustness tester for java. Softw. Pract. Exp. 34(11), 1025-1050 (2004)

7. Fraser, G., Arcuri, A.: Evosuite: Automatic test suite generation for object-oriented software. In: Proc. ESEC/FSE. pp. 416-419. ACM (2011)

8. Fraser, G., Arcuri, A.: The seed is strong: Seeding strategies in search-based software testing. In: Proc. ICST. pp. 121-130. IEEE Comp. Soc. (2012)

9. Fraser, G., Arcuri, A.: Whole test suite generation. IEEE Trans. Software Eng. 39(2), 276-291 (2013)

10. Fraser, G., Arcuri, A.: Automated test generation for java generics. In: Proc. SWQD. LNBPI, vol. 166, pp. 185-198. Springer (2014)

11. Levenshtein, V.I.: Binary codes capable of correcting deletions, insertions, and reversals. In: Soviet physics doklady. vol. 10, pp. 707-710 (1966)

12. Li, G., Andreasen, E., Ghosh, I.: SymJS: Automatic symbolic testing of JavaScript web applications. In: Proc. FSE. pp. 449-459. ACM (2014)

13. Ma, L., Artho, C., Zhang, C., Sato, H., Gmeiner, J., Ramler, R.: Grt: Programanalysis-guided random testing (t). In: Proc. ASE. pp. 212-223. IEEE Comp. Soc. (2015)

14. Mirshokraie, S., Mesbah, A., Pattabiraman, K.: JSEFT: Automated javascript unit test generation. In: Proc. ICST. pp. 1-10. IEEE Comp. Soc. (2015)

15. Pacheco, C., Lahiri, S.K., Ernst, M.D., Ball, T.: Feedback-directed random test generation. In: Proc. ICSE. pp. 75-84. IEEE Comp. Soc. (2007)

16. Panichella, A., Kifetew, F.M., Tonella, P.: Automated test case generation as a many-objective optimisation problem with dynamic selection of the targets. IEEE Trans. Software Eng. 44(2), 122-158 (2018)

17. Sakti, A., Pesant, G., Guéhéneuc, Y.G.: Instance generator and problem representation to improve object oriented code coverage. IEEE Trans. Software Eng. 41(3), 294-313 (2014)

18. Selakovic, M., Pradel, M., Karim, R., Tip, F.: Test generation for higher-order functions in dynamic languages. Proc. ACM Program. Lang. 2(OOPSLA), 161:1161:27 (2018)

19. Tonella, P.: Evolutionary testing of classes. In: Proc. ISSTA. pp. 119-128. ACM (2004) 